\title{
Ultrasensitive Ethene Detector Based on a Graphene-Copper(I) Hybrid Material
}

\author{
Wangyang Fu, ${ }^{\dagger, \S}$ Thomas F. van Dijkman, ${ }^{\ddagger}, \S$ Lia M. C. Lima, ${ }^{\dagger}$ Feng Jiang, ${ }^{\ddagger}$ Grégory F. Schneider, ${ }^{*}, \dagger$
} and Elisabeth Bouwman**;

\footnotetext{
${ }^{\dagger}$ Supramolecular and Biomaterials Chemistry and ${ }^{\ddagger}$ Metals in Catalysis, Biomimetics, and Inorganic Materials, Leiden University, Leiden Institute of Chemistry, P.O. Box 9502, 2300 RA Leiden, The Netherlands
}

Supporting Information

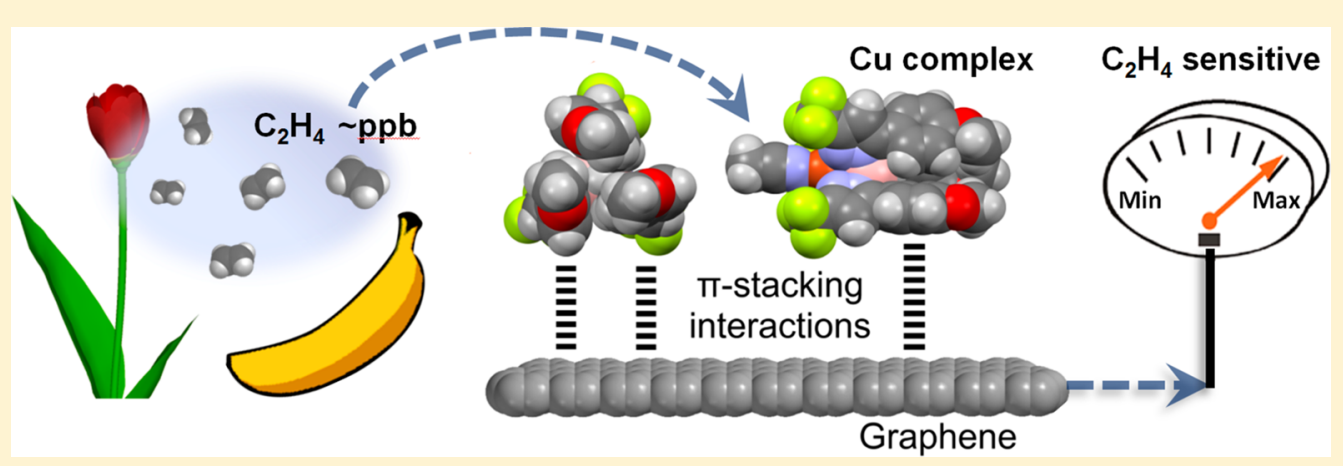

ABSTRACT: Ethene is a highly diffusive and relatively unreactive gas that induces aging responses in plants in concentrations as low as parts per billion. Monitoring concentrations of ethene is critically important for transport and storage of food crops, necessitating the development of a new generation of ultrasensitive detectors. Here we show that by functionalizing graphene with copper complexes biologically relevant concentrations of ethene and of the spoilage marker ethanol can be detected. Importantly, in addition these sensors provide us with important insights into the interactions between molecules, a key concept in chemistry. Chemically induced dipole fluctuations in molecules as they undergo a chemical reaction are harvested in an elegant way through subtle field effects in graphene. By exploiting changes in the dipole moments of molecules that occur upon a chemical reaction we are able to track the reaction and provide mechanistic insight that was, until now, out of reach.

KEYWORDS: Graphene, copper(I) complex, reaction mechanism, dipole moment, ultrasensitive detector

T he plant-hormone ethene has long been known to be essential for the ripening of many fruits. ${ }^{1,2}$ Ethene is also known for its deleterious effects on plant physiology when its concentration builds up, leading to over-ripeness and spoilage in sensitive food crops. ${ }^{3}$ Control of ethene concentrations during transport and storage of crops is therefore crucial, which demands the development of sensitive detectors, particularly sensory systems that show good selectivity for ethene and enable following the ripening and senescing processes in various crops over time. In the past few years, the use of copper(I) compounds (or other similar organometallic complexes) for selective ethene detection has been reported with the detection method based on either optical sensing in combination with fluorescent polymers ${ }^{4}$ or electrical sensing in combination with carbon nanotubes. ${ }^{5}$ However, for potential large-scale applications of such sensors in greenhouses and transportation the sensitivity and/or reproducibility of such sensing devices need further attention. In order to meet the challenges of developing reliable detectors with high sensitivity and selectivity, it is necessary to acquire a fundamental understanding of the chemistry leading to a response, a need that coincides with a core aspect of chemistry as a science, which is the desire to fundamentally understand the interactions between molecules.

A sensor produces a signal when a chemical or physical interaction between a sensitizer and an analyte molecule occurs. The intensity of the response will depend on the reaction of the analyte with the sensitizer and must be correlated to the reaction rate and binding constant of this reaction. Unfortunately, the determination of equilibrium binding constants using currently available bulk techniques is difficult to apply for thin films, hence the need for in situ sensors.

Dipole moments are a measure of the distribution of electron density in a molecule and represent a simplified view of the electrical field surrounding a molecule. The quantification of changes in dipole moments upon a chemical reaction has hardly been exploited in analytical chemistry, including the latest sensing devices and techniques. So far, the use of dipole moments for sensing has been hampered by the subtlety of the

Received: October 20, 2017

Revised: November 28, 2017

Published: November 28, 2017 
effects combined with the lack of sufficiently good signal-tonoise ratios in conventional electronic devices.

Compared to the electrical fields surrounding ions, the fields that surround neutral molecules are typically much weaker and therefore more difficult to detect. ${ }^{7}$ To sense subtle changes in molecular dipole moments over the course of a chemical reaction, a macromolecular sensor with high innate electrical sensitivity is required. A material well-known for its sensitivity to external electrical fields is graphene, the single layer hexagonal carbon allotrope. 8 A striking example of the extreme sensitivity of graphene is the capability of detecting single molecules, ${ }^{10,11}$ even in a highly dilute gas. ${ }^{12}$

Now, we report a novel copper(I)-graphene hybrid material that we employed to study the thermodynamics and kinetics of a chemical reaction using the intrinsic changes in the dipole moments of molecules during the reaction. This allowed us to determine binding constants and reaction rate constants that would otherwise be impossible to obtain.

We previously reported a series of copper(I) complexes with electronic properties that can be varied deliberately. ${ }^{13}$ In this work we report a systematic study of the reactions between ethene or ethanol and these copper(I) complexes of the fluorinated hydridotrispyrazolylborate ligand series $\left[\mathrm{Tp}^{\mathrm{CF} 3,4-\mathrm{RPh}}\right]^{-}$(Figure 1a). The use of such electronically tunable ligands provides systematic influence over the direction and magnitude of the dipole moments of the copper compounds. We measure the changes in these molecular probes upon reaction with ethene or ethanol using graphene field-effect transistors (GFETs). On the basis of the observed rate constants and binding affinities, we formulate a reaction mechanism for the interaction between the copper probes in the hybrid material and the molecules ethene and ethanol. We show that the GFETs can be used to detect ethene and ethanol at biologically relevant concentrations, down to low part-perbillion (ppb) levels.

Copper(I) complexes are known to have strong affinity for ethene in particular. Swager et al. exploited the ability of copper(I) complexes to bind ethene by drop casting singlewalled carbon nanotubes (SWCNTs) onto electrodes together with the fluorinated copper(I) hydridotrispyrazolylborate complex also used in a chemoluminescent sensor. ${ }^{5}$ When the resulting devices were exposed to dilute ethene gas in dry dinitrogen atmosphere reversible changes in their resistivity were observed for ethene concentrations down to $500 \mathrm{ppb}$, a significant improvement over the chemoluminescent sensor $(1000 \mathrm{ppm})$ reported earlier by Swager et al. ${ }^{4}$ The chemiresistive sensor shows reasonable sensitivity, good selectivity and good response times. However, the desirable properties of the sensor are offset by the poor reproducibility due to the use of the SWCNTs as well as the inhomogeneous crystallites of the complex interspersed in between the SWCNTs, which form networks with unpredictable and unstable electrical junctions between the nanotubes. In addition, in order to respond to varying ethene concentrations and prevent spoilage or to induce ripening, detection of ethene at the low range of biologically relevant concentrations $(<100$ $\mathrm{ppb})$ is required, which poses a technological challenge. In contrast, the use of graphene instead of SWCNTs allows for the exploitation of the all-surface-atom makeup offered by carbon allotropes like graphene and SWCNTs without the aforementioned practical problems.

We designed copper(I) complexes based on a common molecular structure with modifications that allow us to control
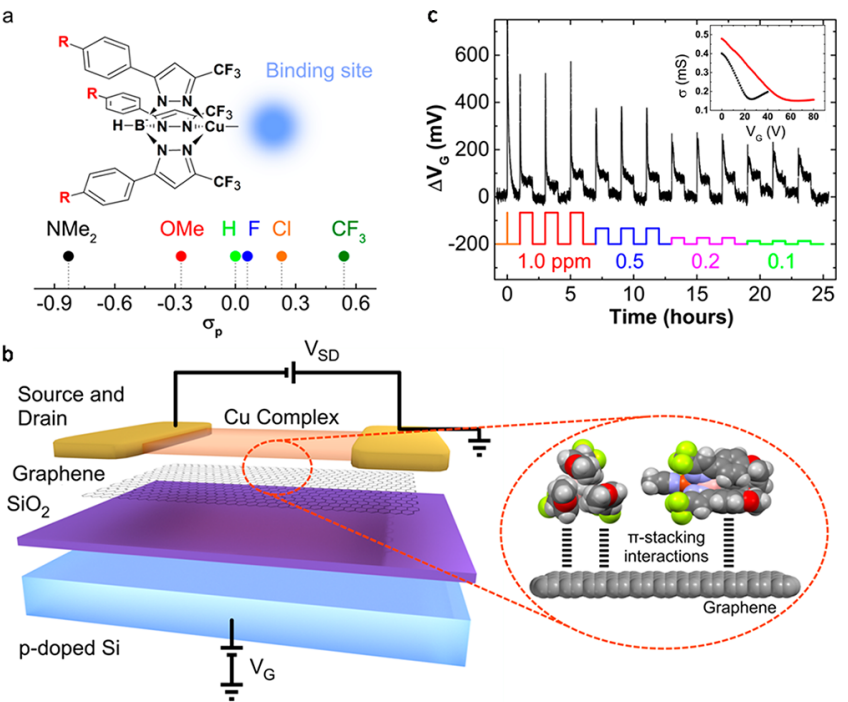

Figure 1. Device layout and concept. (a) Chemical structure of the sensitizer molecules with the substituents " $R$ " in red and the analyte binding site in blue. The substituents $R$ are arranged versus their Hammett $\sigma_{\mathrm{p}}$ parameters. (b) Schematic representation of the GFET device layout. On top of the electrically insulating silicon dioxide layer of a highly $\mathrm{p}^{+}$-doped silicon wafer the substrate graphene is covered in a thin layer of a copper complex. Gold source and drain electrodes complete the electrical circuit through which a potential $\left(V_{\mathrm{SD}}\right)$ is applied. Zoom: exploded view of two space-filling projections of the acetonitrile adduct of the complex with $R=$ OMe shown along the boron-copper axis (left) and side-on (right) as it stacks on graphene. (c) Baseline-corrected trace of the response of a GFET $(R=F)$ exposed to various concentrations of ethene gas. A short initial "scrubbing" exposure of $20 \mathrm{ppm}$ (orange) is followed by $1 \mathrm{ppm}$ (red), $0.5 \mathrm{ppm}$ (blue), $0.2 \mathrm{ppm}$ (purple), and $0.1 \mathrm{ppm}$ (green) exposures, each in triplicate. Switching on the mass-flow controllers causes the initial sharp spikes, which last several seconds. While retaining the applied ethene partial pressure the signal drops within several minutes before it becomes stabilized at a new "baseline" (at $\Delta V_{\mathrm{G}} \sim 80 \mathrm{mV}$, retained for $\sim 1 \mathrm{~h}$ ) as the system reaches equilibrium. Upon switching the gas flow to "air" the signal relaxes to the initial baseline; this desorption process is used in the determination of the dissociation constants. We used the difference between the two baselines (upon ethene exposure) as the measure of the sensing response (instead of the magnitude of the initial spike signal, which is less reliable). Inset: the back gate voltage $\left(V_{\mathrm{G}}\right)$ dependent conductance $(\sigma)$ of graphene on OTS modified $\mathrm{SiO}_{2} / \mathrm{Si}$ substrate before (black) and after (red) the complex $(R=\mathrm{F})$ was applied. The back gate potential $V_{\mathrm{G}}$ is kept at $0 \mathrm{~V}$ during sensing experiments.

the polarity of the complex while leaving the binding pocket for the molecules unaffected (see the Methods and Supporting Information). The ligands are functionalized with trifluoromethyl groups, which help to stabilize copper in its monocationic state. By surrounding the binding pocket with trifluoromethyl groups a size limit is imposed on the molecules that can bind to the copper ion: small molecules such as ethene and ethanol fit while larger molecules such as toluene do not. Phenyl rings were included in the ligands to induce $\pi$-stacking interactions that enhance the electronic coupling between the sensitizer molecules and graphene.

The polarity of the complexes is determined by the interplay between the electron-withdrawing trifluoromethyl groups that surround the binding pocket and the substituted phenyl rings on the other side of the hydridotrispyrazolylborate ligands. A strongly electron-donating substituent leads to a large dipole 
moment due to the "push-pull" effect: electrons pushed into the ligand by the donating group are pulled further by the trifluoromethyl groups. Conversely, an electron-withdrawing substituent counteracts the pull of the trifluoromethyl group resulting in a small dipole moment. The different polarities based on the substituents $R$ are quantified with the Hammett parameter $\sigma_{\mathrm{p}}$; a positive value of the Hammett parameter indicates an electron-withdrawing substituent while a negative value denotes an electron-donating substituent. By judicious choice of the $R$ groups copper(I) compounds with tunable dipole moments were obtained.

The complexes have a coordination site that can be used for reversibly binding a single ligand; we used the synthetically convenient acetonitrile adducts as starting points. In a previous study, the ligand series was shown to form complexes with nearly identical binding pockets. ${ }^{13}$ The X-ray crystal structure of the acetonitrile adduct of the complex with $R=\mathrm{OMe}$ indeed showed the anticipated conformation (see Figure S1). The spectroscopic data of the other acetonitrile adducts conformed to expectations.

GFET devices were prepared by transfer of chemical vapor deposition (CVD) graphene onto highly $\mathrm{p}^{+}$-doped silicon substrates with $285 \mathrm{~nm}$ silicon dioxide insulator layers. Electrodes $(5 \mathrm{~nm} \mathrm{Cr} / 50 \mathrm{~nm} \mathrm{Au})$ were applied directly on top of the graphene to ensure good mechanical stability and negligible contact resistance (Figure $1 \mathrm{~b}$ ). Prior to surface functionalization, we tested the gate voltage $V_{\mathrm{G}}$-dependent sheet resistance of the graphene and plotted the conductance/ voltage curve (Figure 1c, inset). Owing to the trap states at the graphene $/ \mathrm{SiO}_{2}$ surface, ${ }^{14}$ graphene devices fabricated on a $\mathrm{SiO}_{2} / \mathrm{Si}$ substrate are often haunted by intensive $\mathrm{p}^{+}$-doping and large hysteresis. The resulting Dirac points were typically too high to sweep without risking damage to the devices. Therefore, graphene was transferred onto surfaces treated with octadecyltrichlorosilane (OTS) to shield the graphene from trapped charges on the $\mathrm{SiO}_{2}$ surface, resulting in Dirac points closer to 0 $\mathrm{V}$ and hysteresis-free operation (Figure S8). Notably, the Dirac point is still away from $V_{\mathrm{G}}=0 \mathrm{~V}$. Therefore, the GFET exhibits high transconductance and high sensitivity at this set point. The $\sigma\left(V_{\mathrm{G}}\right)$ curves of these GFETs show rather symmetric shapes and high field-effect mobilities of $\sim 1500 \mathrm{~cm}^{2} \mathrm{~V}^{-1} \mathrm{~s}^{-1}$ for hole carriers, which are reduced to approximately $80 \%$ of their initial values upon the functionalization with the copper(I) complexes irrespective of the substituent $R$ in the complex. The drop in charge mobility is ascribed to increased scattering of the charge carriers. ${ }^{15}$ This trend is demonstrated in the inset of Figure 1c: the black and red points are from a GFET on OTS treated $\mathrm{SiO}_{2} / \mathrm{Si}$ substrate before and after the complex $(R=\mathrm{F})$ was applied, respectively. Notably, we observed no trend in the change of the Dirac points before and after the functionalization with different complexes, which indicates that differences in scattering rates caused by differences in surface organization of the complexes obscure the more subtle effect of the different polarities of the complexes.

On the basis of our previous experiences on graphene sensor design $^{8}$ and other reports, ${ }^{16}$ it is known that the reliable operation of a GFET sensor is highly dependent on the formation of homogeneous and ideally a thin layers of a sensitizer on the graphene surface. We speculate that a thin sensitive layer facilitates gas diffusion and allows close-tosurface sensing to deliver fast response times and improved sensitivity. The desired full-surface coverage of the graphene with the copper complexes was obtained by dip-coating pristine
GFET devices in concentrated solutions of the complexes forming thin, uniform layers. Longer incubation periods did not result in a larger shift of the $\sigma / V$-curve, indicating saturation of the $\mathrm{Cu}$ complex adsorbed on the graphene. Our ellipsometry study on dip-coated graphene samples indicated an adsorption density in the order of 5-8 copper complexes per square nanometer corresponding to 3-5 layers coverage (Figure S2). In order to check the stability of the complexes upon dropcasting and exposure to ethene, Raman spectra of the complexes were recorded $(R=\mathrm{OMe}$ with $\mathrm{MeCN}$ and $\mathrm{CO}$ ligands) in bulk crystalline material and on the surface of graphene after drop-casting and self-assembly and after 4 days of storage in ethene (20 ppm). In Figure S7, the Raman spectra are shown, which confirm the presence and stability of the selfassembled layers of complexes on graphene. ${ }^{17}$ As a further check on the stability of the sensors, we have monitored the sensing responses (to ethene at $1 \mathrm{ppm}$ ) of the devices with complexes " $R=\mathrm{OMe}$ " and " $R=\mathrm{CF}_{3}$ " over a testing period of 12 days (Figure S9). The sensing behavior of the devices is stable over these 12 days (within a maximum deviation of $\Delta V_{\mathrm{G}} / \Delta V_{\mathrm{G} 0}<40 \%$ ), indicating the stability of the copper(I) complexes in air. Particularly, we found that the sensitivity of the devices reached a stable level after 4 days of testing (and up to 12 days; Figure S9). Extrapolation of the stability curves in Figure S9 indicates that the designed sensors should be stable in air for a month or longer.

As the GFET surface comprises a discrete number of $\mathrm{Cu}$ complexes as potential binding sites for analyte molecules in a quasi-two-dimensional arrangement it can be described with a Langmuir adsorption isotherm. An important prerequisite for the application of Langmuir isotherms is the reversibility of the underlying chemical reaction, which in this work is confirmed by the return to the baseline of the observed signals. In Figure 1c the baseline-corrected trace is depicted of the $\Delta V_{\mathrm{G}}$ response (in black) of a GFET ( $R=\mathrm{F}$ ) when exposed to ethene pulses (see Methods). The reaction is assumed to involve the conversion of a complex without associated ethene or ethanol (the "off" state) to a complex coordinated with ethene or ethanol (eq 1; the "on" state with association rate constant $k_{1}$ and the dissociation rate constant $k_{-1}$ respectively, "gas" is ethene or ethanol).

$$
\left[\mathrm{Cu}^{\mathrm{I}}\left(\mathrm{Tp}^{\mathrm{CF} 3,4 \mathrm{R}-\mathrm{Ph}}\right)(\text { free site })\right]+\text { gasf-state } \underset{\mathrm{k}_{-1}}{\stackrel{\mathrm{k}_{1}}{\rightleftarrows}}\left[\mathrm{Cu}^{\mathrm{I}}\left(\mathrm{Tp}_{\text {on-state }}^{\mathrm{CF3}, 4 \mathrm{R}-\mathrm{Ph}}\right)(\mathrm{gas})\right]
$$

The results of the gas-sensing experiments were interpreted quantitatively by fitting Langmuir adsorption isotherms to the $\Delta V_{\mathrm{G}} / p_{\text {gas }}$ plots (where $p_{\text {gas }}$ is the partial pressure of the analyte gas in ppm) and can be described using eq 2 .

$$
\Delta V_{\mathrm{G}}=\frac{D_{\mathrm{n}^{\perp}}}{\varepsilon}[\text { on }]_{\max } \times \frac{p_{\text {gas }}}{p_{\text {gas }}+K_{\mathrm{D}}}
$$

Eq 2 describes the change of the signal $\Delta V_{\mathrm{G}}$ upon coordination of the copper(I) center with ethene or ethanol as a function of the surface coverage of graphene. The prefactor $\frac{D_{\mathrm{n}} \perp}{\varepsilon}$ represents the change in the effective charge on graphene that is caused by the conversion of complexes in the "off" state to complexes in the "on" state using a parallel-plate capacitor model; $D_{n}{ }^{\perp}$ represents the out-of-plane polarization density of the aggregated dipole moments of the $\mathrm{Cu}$ complexes and $\varepsilon$ is the dielectric constant of the thin $\mathrm{Cu}$ complex layer. This prefactor multiplied by the maximum surface coverage with complexes in 

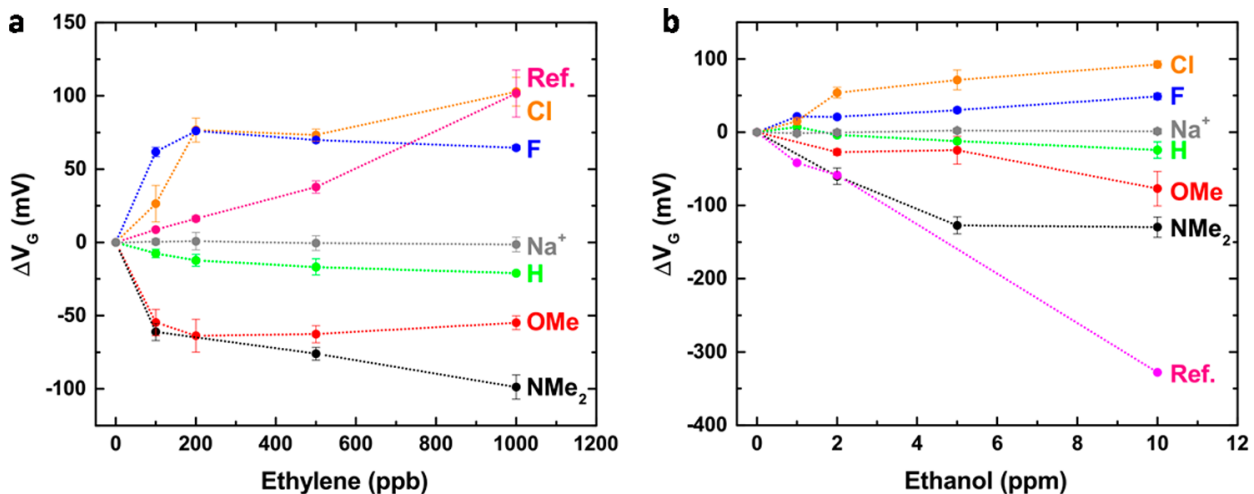

Figure 2. GFET sensor responses. (a) $\Delta V_{\mathrm{G}} / p_{\text {gas }}$ plots of the GFET sensors comprising the sensitizing complexes $\left[\mathrm{Cu}\left(\mathrm{Tp}{ }^{\mathrm{CF} 3,4-\mathrm{RPh}}\right)(\mathrm{MeCN})\right](R=$ $\mathrm{NMe}_{2}, \mathrm{OMe}, \mathrm{H}, \mathrm{F}$, and $\left.\mathrm{Cl}\right),\left[\mathrm{Cu}\left(\mathrm{Tp}^{(\mathrm{CF} 3) 2}\right)(\mathrm{MeCN})\right]$ ("Ref.", pink), or $\mathrm{NaTp}^{\mathrm{CF} 3, \mathrm{Ph}}$ ("Na", gray) upon exposure to different concentrations of ethene gas. $R=\mathrm{CF}_{3}$ is omitted as all devices functionalized with this compound showed insufficient signal-to-noise ratios and only the highest analyte concentrations (1.0 ppm for ethene and $10.0 \mathrm{ppm}$ for ethanol) could be detected reliably. Error bars indicate standard errors. (b) The same plot as (a) but for the ethanol exposures. Note the different scales on the horizontal axis.

the "on" state $[\text { on }]_{\max }$ gives the maximum amplitude of the signal and is effectively a scalar for the Langmuir adsorption isotherm. The second term is the Langmuir adsorption isotherm that describes the degree to which the graphene surface is covered by complexes in the "on" state as a function of the partial pressure of the analyte gas (" $p_{\text {gas }}$ ") and the equilibrium dissociation constant $K_{\mathrm{D}}$. Fitting plots of concentration versus response intensities with eq 2 offers the possibility to extract the equilibrium dissociation constant $K_{\mathrm{D}}$ and the prefactor $\left(\frac{D_{\mathrm{n}^{\perp}}}{\varepsilon}\right)[\text { on }]_{\max }$. When working with gases, $K_{\mathrm{D}}$ is most conveniently expressed using a molar fraction (i.e., ppm or $\mathrm{ppb}$ ).

Aside from the equilibrium dissociation constant $K_{\mathrm{D}}$, the (first-order) dissociation rate constant $k_{-1}$ of desorption of the analyte gases can be extracted from the traces of the gas sensing experiments. When fitting the dissociation phase of sensing experiments, care must be taken to consider both the dissociation and any possible reassociation of the analyte, as molecules require time to be flushed away from the surface: the removal of the gas is best described using a half-life based on the rate at which the complete internal volume of the flow cell is displaced. The flow rate was therefore set high enough to completely displace the internal volume of our flow cell in approximately three seconds, a rate at which the analyte concentration dropped below detectable levels long before the output signals returned to the baseline. ${ }^{18}$ Therefore, the kinetics of the dissociation can be modeled without considering the association rate constant $k_{1}$, leading to a simple exponential equation in which the signal decays from $V_{\text {on }}$, the potential during exposure, to $V_{\text {off }}$ the baseline signal, as expressed by eq 3.

$V_{\mathrm{G}}=V_{\text {on }} e^{-k_{-1} t}+V_{\text {off }}$

Upon exposure to ethene all sensors showed saturation below $1.0 \mathrm{ppm}$, except for a device prepared with a complex that has been studied previously on carbon nanotubes (i.e., $\left.\left[\mathrm{Cu}\left(\mathrm{Tp}^{(\mathrm{CF} 3) 2}\right)(\mathrm{MeCN})\right]\right),{ }^{5}$ which in our device showed a linear response in the entire concentration range (Figure 2a,b). Surprisingly, the sensor functionalized with $\left[\mathrm{Cu}\left(\mathrm{Tp}^{(\mathrm{CF} 3) 2}\right)\right.$ $(\mathrm{MeCN})]$ shows signals with opposite signs for ethene and ethanol and shows no signs of saturation even at the highest concentrations. Evidently the $\left[\mathrm{Cu}\left(\mathrm{Tp}^{\left(\mathrm{CF}_{3}\right) 2}\right)\right]$ fragment binds much more weakly to the analyte molecules than our copper compounds. In the case of $R=\mathrm{OMe}$ or $\mathrm{F}$, saturation of the sensors was even observed at $0.1 \mathrm{ppm}$. For ethanol, the devices showed signs of saturation at $10 \mathrm{ppm}$. During testing the gas temperature was constantly monitored, so any spurious signals due to temperature fluctuations were ruled out. We also tested the sensing response of a GFET with an inert sodium salt of the ligand $(R=\mathrm{H})$ upon exposure to ethene $(0.1-1 \mathrm{ppm})$ and ethanol $(1-10 \mathrm{ppm})$ gas: it appeared to respond to neither ethene (Figure 2a) nor ethanol (Figure 2b). Finally, another control experiment with bare graphene showed negligible responses to ethene or ethanol. We thus conclude that the gas sensing responses of our sensors must originate from the presence of the copper(I) complexes.

As the sensitivity of our sensors is very high and thus the required concentrations of the analyte gas are low, drift in the baseline and poor signal-to-noise ratios occasionally obscured the signal as reflected in the error bars. Nonetheless clear trends emerge when the responses of the sensors are reproduced and collated. The complexes with electron-donating ligands $(R=$ $\mathrm{NMe}_{2}, \mathrm{OMe}$, and $\mathrm{H}$ ) produce signals with a negative $\Delta V_{\mathrm{G}}$ whereas the ligands with electron-withdrawing substituents $(R$ $=\mathrm{F}$ and $\mathrm{Cl}$ ) produce signals with a positive $\Delta V_{\mathrm{G}}$, a trend that is clearly visible both for the ethene and ethanol exposures (Figure 2a,b). Remarkably, the amplitude of the signals correlates approximately linearly with the Hammett $\sigma_{\mathrm{p}}$ parameters of the substituents $R$ (Figure 3a). The responses correlate more linearly for the ethanol exposures than in the ethene exposures, particularly for the more polar complexes, indicating subtle differences in binding modes of ethene and ethanol. Contrary to expectations, the sensor comprising the complex with $R=\mathrm{CF}_{3}$ produced a negative signal when exposed to $1.0 \mathrm{ppm}$ ethene gas, whereas ethanol exposures produced the expected positive signals. Ellipsometry data and atomic force microscopy (AFM) images showed no unusual surfaces for the devices with the acetonitrile adduct of the complex $R=\mathrm{CF}_{3}$ compared to those of the other devices (Figure S3-6).

The equilibrium dissociation constants $K_{\mathrm{D}}$ were obtained for a number of the complexes and are clearly distinct for the ethene and ethanol complexes (Figure $3 \mathrm{c}$ ). The $K_{\mathrm{D}}$ values of the ethene complexes are $0.11(3)\left(R=\mathrm{NMe}_{2}\right), 0.23(3)(R=$ $\mathrm{H})$ and $0.21(7) \mathrm{ppm}(\mathrm{R}=\mathrm{Cl})$, whereas the values obtained for the ethanol complexes are considerably higher at 3(1) $(R=$ $\left.\mathrm{NMe}_{2}, \mathrm{~F}\right)$, and $4(1) \operatorname{ppm}(R=\mathrm{Cl})$. Physically, $K_{\mathrm{D}}$ can be 

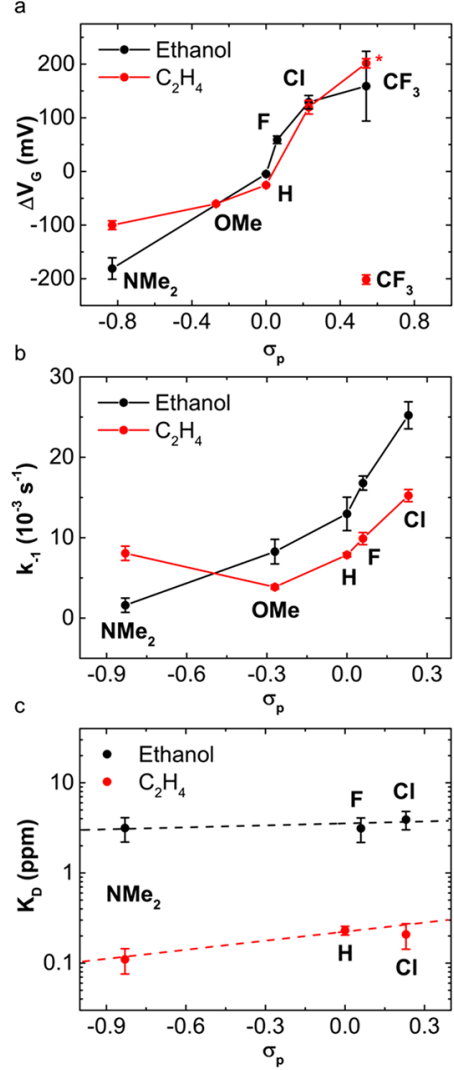

Figure 3. Sensor response and calculated affinity constants. (a) Response intensities (as $\Delta V_{\mathrm{G}}$ in $\mathrm{mV}$ ) at $1.0 \mathrm{ppm}\left(\mathrm{C}_{2} \mathrm{H}_{4}\right)$ and $10 \mathrm{ppm}$ (ethanol) versus the Hammett $\sigma_{\mathrm{p}}$ parameters of the substituents on the ligands of the copper(I) complexes. The ethene response of the device functionalized with the complex $R=\mathrm{CF}_{3}$ marked with $*$ is the actual signal with its sign reversed. (b) First-order dissociation rate constants $\left(k_{-1}\right)$ of the ethanol (black squares) and ethene (red circles) complexes versus the Hammett $\sigma_{\mathrm{p}}$ parameters of the substituents on the ligands. (c) Equilibrium dissociation constants of ethene (red) and ethanol (black) on the GFETs as a function of the Hammett parameter of the substituent on the ligand of the sensitizer.

interpreted as the fraction of the analyte gas at which half of the active copper complexes on the graphene surface is coordinated by analyte molecules. Clearly the GFETs can be exposed to higher concentrations of ethanol than of ethene before becoming saturated. The affinity is much higher for ethene binding than for ethanol, so that the sensor will react to ripening but not much to decay. In order to make this device ready for commercial applications, further development is necessary, for example, the exclusion of the remaining cross sensitivities is a logical point of interest. A potential crosssensitivity could arise for $\mathrm{CO}$, which at this moment we cannot exclude; future work will be directed to study this potential cross-sensitivity. In principle, $\mathrm{CO}$ sensitivity can be overcome using a protective layer on the sensor device. ${ }^{19}$ The equilibrium dissociation constant of $\left[\mathrm{Cu}\left(\mathrm{Tp}^{(\mathrm{CF} 3) 2}\right)\left(\mathrm{C}_{2} \mathrm{H}_{4}\right)\right]$ was not determined as saturation was never reached but must be considerably higher as the response curve shows no signs of saturation at $1.0 \mathrm{ppm}$. Notably, a clear correlation between the electron density on the copper(I) center and the $K_{\mathrm{D}}$ of the ethene and ethanol complexes is not observed. A possible explanation is that the electron-donating and withdrawing interactions upon binding are more or less balanced for a given analyte gas binding at the free site, so that there is no trend with the substituent on the ligand and thus the electron density at $\mathrm{Cu}$.

The dissociation rate constants $\left(k_{-1}\right)$ were obtained from the traces of the dissociation phases of the gas exposure experiments. For the ethanol complexes, $k_{-1}$ continues to decrease with decreasing $\sigma_{\mathrm{p}}$ (Figure $3 \mathrm{~b}$ ), while for the ethene complexes the dissociation rate constants reveal an apparent minimum at $\sigma_{\mathrm{p}}=-0.27(R=\mathrm{OMe})$. With decreasing $\sigma_{\mathrm{p}}$, the $\pi$ backbonding interactions to ethene become larger. As the rate of the dissociation reaction is dependent, among other factors, on the bond dissociation energy of the complexes, one would expect lower dissociation rate constants at lower $\sigma_{\mathrm{p}}$ values. The nonlinearity of the observed dissociation rates with respect to $\sigma_{\mathrm{p}}$ indicates that bond dissociation energies are only part of the total contributions to the dissociation rate constants. Indeed, there appears to be additional, less well understood complexity in the behavior of the different compounds.

It is possible to estimate the detection limit of the new sensors using the best (i.e., smallest) peak-to-peak noise we observed $(1 \mathrm{mV})$ and to extrapolate the performance of sensors comprising the $\mathrm{NMe}_{2}$-substituted complex. Assuming a signalto-noise ratio of at least two is required to identify a signal, the extrapolated lower limits of detection are approximately $2 \mathrm{ppb}$ for ethene and $35 \mathrm{ppb}$ for ethanol. Using standard conditions 1 $\mathrm{ppb}$ corresponds to approximately $41 \mathrm{pM}$ which translates to limits of detection of approximately $82 \mathrm{pM}$ for ethene and 1.4 $\mathrm{nM}$ for ethanol. Using eq 2 we can estimate the surface coverage of the on state complexes at the detection limits which shows that both for ethene and ethanol the detection limit occurs at approximately $1 \%$ surface coverage. This is a relatively high percentage compared to similar protein-functionalized GFETs, which underscores the subtlety of the effects being observed. $^{20,21}$

The electrical responses generated by the GFETs upon analyte exposure are the result of fluctuations in the doping levels of graphene caused by changes in the chemical and physical properties of the copper complexes. Typically in GFETs the strongest signals are generated by direct charge transfer into the graphene by analyte molecules, such as the generation of holes (oxidation) by $\mathrm{NO}_{2} \cdot{ }^{12}$ Charge-charge transfer is also suggested to be the sensing mechanism of $\left[\mathrm{Cu}\left(\mathrm{Tp}^{(\mathrm{CF} 3) 2}\right)\right]$ on carbon nanotubes to ethene gas, ${ }^{5}$ based on a trigonal copper(I) complex in which one of the pyrazole groups dissociated from the copper(I) center in favor of coordination to the carbon nanotubes. However, we believe such a 16electron structure likely to be the result of a local-minimum during calculations (as indicated by the high energy of this intermediate compared to the ethene-bound complex) and thus do not assume the involvement of such a structural rearrangement to play a role in our sensing devices. ${ }^{5}$ In addition, charge transfer from the complexes to graphene would result in a Dirac point shift before and after the functionalization of graphene that is dependent on the Hammett constants $\sigma_{\mathrm{p}}$ of the substituents $R$. The fact that we observed no such trend in the change of the Dirac points before and after the functionalization with the complexes suggests that in our sensors the charge transfer mechanism does not dominate. Instead a more subtle effect such as modulation of the field effect by the dipole moments of the molecules must be responsible for the generated signals. As the dipole moment of a molecule has a direction depending on the geometry of the molecule, any anisotropic arrangement of the molecules on the graphene 
surface will effectively result in the attraction or repulsion of charges in the graphene toward or away from the molecules.

To rationalize the obtained results, we considered the structures of the complex molecules in their on and off states. The structures of the on states, after exposure of the GFETs to ethene, must resemble those of the structures determined with X-ray crystallography. ${ }^{13}$ The structures of the ethanol complexes are not known but can reasonably be assumed to be similar to those of the carbon monoxide or acetonitrile complexes with the oxygen atom of the ethanol molecule coordinated to the copper center. We propose that the off state consists of an energetically favorable symmetric dinuclear complex with a near-zero dipole moment formed from the remaining mononuclear 16-electron complex upon dissociation of the analyte ligand (Figure 4a). Crystal structures of such

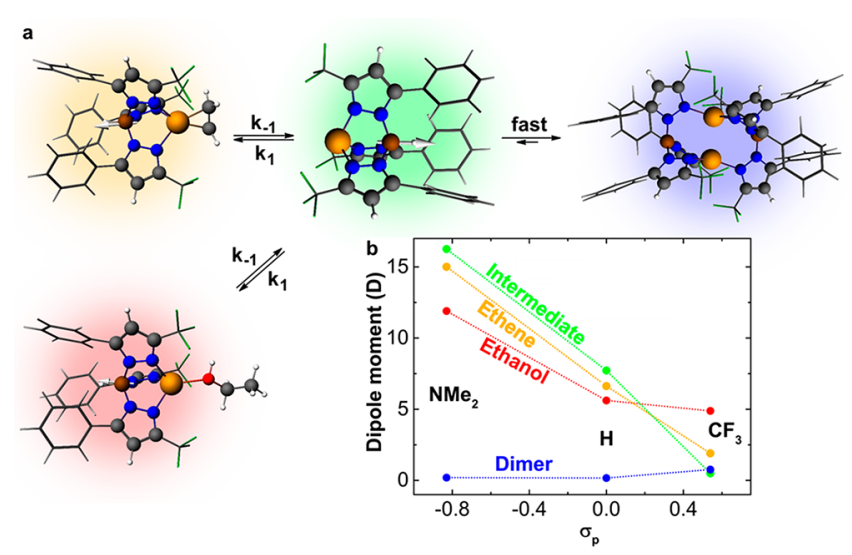

Figure 4. Calculated dipole moments. (a) The reaction mechanism of the surface chemistry on the GFET devices shown using the calculated structures $(R=H)$ of the ethene (orange), ethanol (red), intermediate mononuclear 16-electron (green) and dimeric (blue) complexes after geometry optimization using DFT (ZORA-OPBE/QZ4P, vacuum). (b) Calculated dipole moments versus the Hammett $\sigma_{\mathrm{p}}$ parameters of the substituents $R$ on the ligands.

dimers have been reported for complexes such as $[\mathrm{Cu}$ $\left.\left(\mathrm{Tp}^{\mathrm{CF} 3, \mathrm{Me}}\right)\right]_{2}, \quad\left[\mathrm{Cu}\left(\mathrm{Tp}^{\mathrm{Ph} 2}\right)\right]_{2}, \quad\left[\mathrm{Cu}\left(\mathrm{Tp}^{\mathrm{tBu}}\right)\right]_{2}$, and $[\mathrm{Cu}-$ $\left.\left(\mathrm{Tp} \mathrm{p}^{\mathrm{tBu}, \mathrm{Me}}\right)\right]_{2} \cdot{ }^{22-24}$ Furthermore, it has been reported that in solution the complex $\left[\mathrm{Cu}\left(\mathrm{Tp}^{\mathrm{tBu}, \mathrm{Me}}\right)(\mathrm{MeCN})\right]$ exists in equilibrium with the dimer $\left[\mathrm{Cu}\left(\mathrm{Tp}^{\mathrm{tBu}, \mathrm{Me}}\right)\right]_{2}$; in ${ }^{1} \mathrm{H}$ NMR only the acetonitrile complex and the dinuclear complex were observed but not the mononuclear 16-electron species $\left[\mathrm{Cu}\left(\mathrm{Tp} \mathrm{p}^{\mathrm{tBu}, \mathrm{Me}}\right)\right] .^{24}$ It was therefore concluded that in solution the interconversion between the mononuclear 16-electron species $\left[\mathrm{Cu}\left(\mathrm{Tp}^{\mathrm{t} \mathrm{Bu}, \mathrm{Me}}\right)\right]$ and the dimeric compound $[\mathrm{Cu}-$ $\left.\left(\mathrm{Tp}^{\mathrm{tBu}, \mathrm{Me}}\right)\right]_{2}$ proceeds rapidly and nearly quantitatively. Attempts have been undertaken to image $\mathrm{Cu}(\mathrm{I})$ scorpionate complexes on HOPG using AFM and STM (scanning tunneling microscopy); for these studies, complexes were designed with large naphthalene rings optimized for adsorption onto a graphene surface. Unfortunately the resolution was insufficient to make definitive statements regarding the formation of the proposed dimers. ${ }^{25}$

To gain insight into the influence of the substituents on the ligands on the dipole moments of the copper complexes, the most polar complexes $\left(R=\mathrm{NMe}_{2}\right)$, the least polar complexes $\left(R=\mathrm{CF}_{3}\right)$ and intermediate complexes $(R=\mathrm{H})$ were modeled using density functional theory. The expected trends are clearly visible; as the substituent $R$ becomes more electron-donating, the dipole moments of the complexes increase (Figure $4 b$ ). The deviation from the trend in the ethanol complexes for $R=\mathrm{CF}_{3}$ is due to a distortion in the calculated model caused by a hydrogen bond between the ethanol ligand and one of the fluorine atoms of a $\mathrm{CF}_{3}$ group. Additionally it is important to keep in mind that the exact structures and thus their dipole moments are affected by external forces such as packing effects on graphene and $\pi$-stacking interactions between the complex molecules, which are neglected here. The calculated values do show, however, that the dipole moments of the molecules can be related to the Hammett $\sigma_{\mathrm{p}}$ parameters of the substituents on the ligands, which reinforces the impression that deviations from the trend in the gas-sensing results are most likely due to differences in the exact orientations of the complex molecules on the graphene.

The calculated dipole moments show a clear distinction between the ethene and ethanol complexes having significant dipole moments, and the dinuclear off state complexes that have dipole moments of nearly zero Debye. As evident from DFT calculations the dipole moments of the complexes show little change upon dissociation of the ethene or ethanol ligands. Only when the dinuclear species are formed, the dipole moment becomes nearly zero. This result forms the basis for the hypothesis that the off state consists of the dimeric complexes rather than the mononuclear 16 electron complexes. Thus, the mechanism of sensing we propose is based on the conversion of the polar complexes in the on state to the "apolar" dinuclear complexes in the off state.

In conclusion, we have successfully demonstrated that our hybrid GFETs can be used to probe intermolecular interactions using changes in the dipole moments of the reactants. For the first time, inorganic copper complexes are combined with graphene to build ethene detectors, resulting in a sensor that exhibits reproducible detection of ethene with larger sensitivity (down to $2 \mathrm{ppb}$ ) compared to existing technologies. By using a systematically engineered set of probe molecules we obtained useful information such as reaction rates and equilibrium constants, which were used to derive a plausible reaction mechanism. The use of a Langmuir adsorption isotherm allowed for the extraction of $K_{\mathrm{D}}$ and $k_{-1}$ values, which are physically meaningful data that are difficult to obtain otherwise.

The equilibrium dissociation constants of the ethene and ethanol complexes depend only slightly on the electronic properties of the ligands. In contrast, variations in the amplitudes and the signs of the responses generated by the devices upon exposure to ethene and ethanol were found to scale well with the dipole moments of the complexes. The strong correlation between the dipole moments and the device responses that we found demonstrates that direct charge transfer to and from graphene is by no means the only feasible mechanism by which graphene sensors may generate signals.

The global human population grows rapidly and the need for a stable food supply grows accordingly. A key challenge in a reliable food supply is to avoid ethene-induced spoilage during transport and storage of sensitive crops. The GFET devices shown in this work present a promising platform to study the interactions between molecules as they occur. Furthermore, these devices may be further developed to generate small sensors to be used in the transportation and storage of food crops.

Methods. General Considerations. All manipulations of air-sensitive compounds were performed in an atmosphere of purified argon gas using standard Schlenk techniques. The synthesis of the sodium salts of the ligands was described 
previously. ${ }^{13}$ All solvents were purchased from commercial sources and reagent grade. The graphene used in this work was purchased from Graphenea Inc. Solvents used for air-sensitive manipulations were dried and deaerated using a PureSolv MD 5 Solvent Purification System and stored on $3 \AA$ molecular sieves under argon. When appropriate, glassware was flame-dried in vacuo immediately prior to use. NMR spectra were recorded on a Bruker AV500 spectrometer $\left(500 \mathrm{MHz}\right.$ for ${ }^{1} \mathrm{H}, 471 \mathrm{MHz}$ for ${ }^{19} \mathrm{~F}$ and $126 \mathrm{MHz}$ for ${ }^{13} \mathrm{C}$ ). Elemental analyses were performed by the Microanalytical laboratory Kolbe in Germany. IR spectra were recorded on a PerkinElmer UATR Two FT-IR spectrometer set to a resolution of $1 \mathrm{~cm}^{-1}$. ESI-MS spectra of compounds in $\mathrm{MeCN}$ were recorded on a Thermal Finnigan AQA ESI-MS system. Contact angles were determined using a Ramé-Hart goniometer using drops of milli-Q water. Multiple drops were used and the results averaged. Ellipsometry was performed using a WVase Ellipsometer from J. A. Woollam Co. Inc. and fitted using a Cauchy model. Data analysis was performed using Origin 9.1 (OriginLab). AFM experiments were performed using a Nanoworld USC-FO.3-KO.3 tip in a JPK NanoWizard Ultra Speed AFM. Calculations were performed with the Amsterdam Density Functional (ADF) software at the ZORA-OPBE/QZ4P level of theory in vacuum using the crystal structures of $\left[\mathrm{Cu}\left(\mathrm{Tp}^{\mathrm{CF} 3,4 \mathrm{R}-\mathrm{Ph}}\right)\left(\mathrm{C}_{2} \mathrm{H}_{4}\right)\right](R=$ $\mathrm{NMe}_{2}, \mathrm{H}$ and $\left.\mathrm{CF}_{3}\right)$ and $\left[\mathrm{Cu}\left(\mathrm{Tp}^{\mathrm{Ph} 2}\right)\right]_{2}$ or modified versions thereof as the initial structures. $13,22,26$

Lock-in Technique. When used for ultrasensitive detection, the resistance change of the GFETs might be very small and overwhelmed by noise. In order to recover the very weak (and in our case slow) sensing signal, we employed a lock-in amplifier (HF2LI, Zurich Instruments) to measure with very narrow bandpass filters $(\sim 1 \mathrm{~Hz})$. We used the HF2LI to generate a sinusoidal alternating voltage with amplitude $\left(V_{\mathrm{SD}} \sim\right.$ $10 \mathrm{mV}$ ) applied to the source and drain electrodes of the GFETs. The resultant source-drain current $I_{\mathrm{SD}}$ across the GFETs (proportional to graphene conductance $\sigma=I_{\mathrm{SD}} / V_{\mathrm{SD}}$ ) was monitored versus time at a bandwidth of $\sim 1 \mathrm{~Hz}$ using the ZiControl (Zurich Instruments) program. Any changes in the GFET conductance $\Delta \sigma$ upon ethene or ethanol gases can be directly related to its gate voltage shift $\Delta V_{\mathrm{G}}$ using the transconductance $g_{\mathrm{m}}\left(\Delta \sigma=g_{\mathrm{m}} \Delta V_{\mathrm{G}}\right)$ obtained from the inset of Figure $1 \mathrm{c}$ at $V_{\mathrm{G}}=0$. A noise frequency sweep was performed before every measurement to identify the testing frequencies with minimum noise power spectrum density and thus optimizing the signal-to-noise ratio. A temperature sensor (Pt100) was mounted at the outlet of the gas tube and the gas temperature could be read off in situ (experiments were conducted at room temperature).

Silanization of the Wafer Substrates. Si wafers with $285 \mathrm{~nm}$ $\mathrm{SiO}_{2}$ were cleaned by rinsing with 2-propanol and milli-Q water. After being blown dry, the substrates were immersed in a warm piranha (mixture of $\mathrm{H}_{2} \mathrm{SO}_{4}$ and $\mathrm{H}_{2} \mathrm{O}_{2}$ ) solution for at least $60 \mathrm{~min}$, rinsed with deionized water, and dried at $150{ }^{\circ} \mathrm{C}$ for $1 \mathrm{~h}$. Thus, cleaned, hydrophyllized, and dried the substrates were immersed in a $10 \%$ solution of trimethoxyoctadecylsilane (OTS, Sigma-Aldich, 90+\%) in hexane and incubated at $60{ }^{\circ} \mathrm{C}$ overnight. For trimethylsilane (TMS) modification, TMSCl was used instead in combination with a few drops of ethyldiisopropylamine. The following day the substrates were rinsed sequentially using hexane, toluene, ethanol, and water before being heated at $110{ }^{\circ} \mathrm{C}$ for at least $1 \mathrm{~h}$. The quality of the surface modifications was verified by sessile drop contact angle measurements which showed contact angles of $\sim 100^{\circ}$ for the OTS-modified surfaces and $\sim 84^{\circ}$ for TMS-modified surfaces.

Graphene Deposition. The transfer of the chemical vapor deposition graphene films from $\mathrm{Cu}$ film to the substrate was done by first spin-coating a PMMA (poly(methyl methacrylate)) layer over the graphene film on copper. ${ }^{27}$ After etching away by oxygen plasma any graphene that was not covered by the PMMA, the $\mathrm{Cu}$ film was dissolved in an ammonium persulfate solution $(0.5 \mathrm{M})$. The solution was then completely exchanged multiple times with milli-Q water to remove as much of the dissolved salts as possible. Eventually, the graphene together with the polymer film was left floating in the aqueous phase from which it is carefully scooped up using a $\mathrm{SiO}_{2} / \mathrm{Si}$ substrate. The PMMA film was dissolved using acetone, leaving uniform, large area monolayer graphene on the substrate for further processing. In order to remove residues left behind during the final washing step, the graphene can be further cleaned by annealing in forming gas $\left(8: 2 \mathrm{Ar} / \mathrm{H}_{2}, 1-10 \mathrm{mbar}, 80\right.$ sccm flow) at $350{ }^{\circ} \mathrm{C}$ for $1 \mathrm{~h}$. In case OTS-modified substrates were used, milder annealing conditions $\left(160{ }^{\circ} \mathrm{C}, 1 \mathrm{~h}\right)$ were used to preserve the OTS layers.

Device Construction. Silicon substrates with graphene and $\mathrm{Au}$ electrodes were immersed for $10 \mathrm{~min}$ in $10 \mathrm{mM}$ dichloromethane solutions of the copper complexes. The samples were then extensively rinsed using a stream of pure dichloromethane from a syringe before being blown dry in a stream of argon (Linde gas, $4.6 \mathrm{~N}$ ) filtered through PTFE filter (pore size $0.45 \mu \mathrm{m}$ ) to exclude dust. The samples were annealed at $50{ }^{\circ} \mathrm{C}$ for $10 \mathrm{~min}$ and then immediately installed in the flow cell and flushed with air $(200 \mathrm{sccm})$ for several hours to stabilize drift.

Characterization of the Copper(I) Layers [Cu$\left.\left(T p^{C F 3,4 R-P h}\right)(\mathrm{MeCN})\right]$. Using ellipsometry the thickness of the layers was studied and was found to range from $2.0(1) \mathrm{nm}$ in the case of $\left[\mathrm{Cu}\left(\mathrm{Tp}^{\mathrm{CF} 3,4 \mathrm{~F}-\mathrm{Ph}}\right)(\mathrm{MeCN})\right]$ to $3.7(1) \mathrm{nm}$ for $\left[\mathrm{Cu}\left(\mathrm{Tp}^{\mathrm{CF} 3,4 \mathrm{CF} 3-\mathrm{Ph}}\right)(\mathrm{MeCN})\right]$. The use of $[\mathrm{Cu}-$ $\left.\left(\mathrm{Tp}^{\mathrm{CF} 3,4 \mathrm{~F}-\mathrm{Ph}}\right)(\mathrm{MeCN})\right]$ was found to result in the thinnest layers but is less suitable for comparison with the other samples as the solubility of $\left[\mathrm{Cu}\left(\mathrm{Tp}^{\mathrm{CF} 3,4 \mathrm{~F}-\mathrm{Ph}}\right)(\mathrm{MeCN})\right]$ in dichloromethane is too low to reach the desired concentration of 10 $\mathrm{mM}$ used for dip-coating of the other compounds. If $\left[\mathrm{Cu}\left(\mathrm{Tp}^{\mathrm{CF} 3,4 \mathrm{~F}-\mathrm{Ph}}\right)(\mathrm{MeCN})\right]$ is excluded from the series, the thinnest layer observed was found for $\left[\mathrm{Cu}\left(\mathrm{Tp}^{\mathrm{CF} 3, \mathrm{Ph}}\right)(\mathrm{MeCN})\right]$ at 2.46(9) $\mathrm{nm}$. The layer thicknesses do not appear to correlate with the polarity of the complexes; rather it appears that properties such as the steric bulk of the complexes and differences in their packing on the graphene surface are responsible. The layer thicknesses correspond to multiple times the height of a monolayer assuming the complexes adsorb sideon. For example, using the crystal structure of $[\mathrm{Cu}-$ $\left.\left(\mathrm{Tp}^{\mathrm{CF} 3,4-\mathrm{OMePh}}\right)(\mathrm{MeCN})\right]$ the thickness of a monolayer was estimated to be approximately $0.8 \mathrm{~nm}$, the layer on graphene of this compound was determined to be $3.31(8) \mathrm{nm}$ thick amounting to approximately 4 monolayers. AFM was used to study the topography of the devices; they appeared flat with small rippling features covering the surface. Upon exposure of a few of the devices to ethene, the surfaces appeared to smooth out somewhat, likely as a result of loss of crystallinity when a mixture of compounds is formed after exposure to the ethene. The smoothing effect was most prominent in the device functionalized with $\left[\mathrm{Cu}\left(\mathrm{Tp}^{(\mathrm{CF} 3) 2}\right)(\mathrm{MeCN})\right]$ which is the most "Teflon-like" molecule and thus least prone to crystallization. This is probably because the acetonitrile ligand can be 
"scrubbed" from the system by a first exposure of the devices to a high concentration $(20 \mathrm{ppm})$ of ethene after which all devices showed reversible responses to ethene.

Gas Detection Experiments. The GFET devices were mounted on gastight epoxy chip carriers and placed in a Teflon flow cell that was tightly sealed using a poly(dimethylsiloxane) (PDMS) ring. Using two mass-flow controllers (MFCs) ethene gas ( $1 \%$ in synthetic air composed of $79 \% \mathrm{~N}_{2}$ and $21 \% \mathrm{O}_{2}$ ) was further diluted with synthetic air to reach concentrations of $1,0.5,0.2$, and $0.1 \mathrm{ppm}$, the range within which most climacteric crops respond to ethene exposure. As shown in Figure 1c, the $\Delta V_{\mathrm{G}}$ signals show a sharp initial spike upon exposure to a gas mixture containing ethene, an indicator that the equilibrium at the surface of the sensor is disturbed by a stimulus (caused by possible "supersaturation" of the sensor when switching the mass flow controllers to introduce ethene at various concentrations). The initial spike normally lasts several seconds and drops within several minutes before it becomes stabilized at a new baseline $\left(\Delta V_{\mathrm{G}} \sim 80 \mathrm{mV}\right.$, retained for $\sim 1 \mathrm{~h}$ ) as the system regains equilibrium. The new baseline at equilibrium occurs during ethene exposure and its magnitude was used as the measure of the sensing response. Upon switching the gas flow to "air" the signal relaxes to the original baseline; this desorption process is used to model the dissociations for the determination of the dissociation constants. As ethanol was expected to bind considerably more weakly to the copper(I) centers than ethene, ethanol exposures were performed at 10, 5, 2, and 1 $\mathrm{ppm}$. As initial experiments showed large spurious responses in the presence of atmospheric moisture, all gas measurements were performed using dry gases.

Noise Measurements. The electrical noise represents another challenge in the sensing experiments. Generally, nanometer- or micrometer-sized graphene electronic devices are haunted by the well-known ubiquitous $1 / f$ noise, whose power spectral density shoots up inversely with reducing the frequency $f$. Such $1 / f$ noise therefore dramatically limited the sensing performances of graphene sensor devices at low frequency where most of the chemical reactions take place (millisecond to tens of seconds). Minimizing noise is crucial for weak dipolar signal detection, because the noise level sets the minimum change in dipole fluctuations that can be resolved. In order to achieve high-performance GFET with optimized signal-to-noise ratio, we used large-area millimeter graphene flakes to eliminate low frequency $1 / f$ noise as the $1 / f$ noise scales inversely quadratically with the area; ${ }^{28}$ at the same time, our sensing signal is unchanged compared to nanoscale devices as it is given by the density and the properties (dipoles) of the molecules and is surface area-independent (this is true as long as we are not looking at single molecule adsorption).

\section{ASSOCIATED CONTENT}

\section{S Supporting Information}

The Supporting Information is available free of charge on the ACS Publications website at DOI: 10.1021/acs.nanolett.7b04466.

Supporting Information shows the synthesis and characterizations of the complexes, high-performance GFET on $\mathrm{SiO}_{2} / \mathrm{Si}$ with OTS-SAM functionalization, lifetime of GFET with copper(I) complexes, gate potential due to $2 \mathrm{D}$ array of dipoles, data processing, and DFT-optimized structures (PDF)

\section{AUTHOR INFORMATION}

\section{Corresponding Authors}

*E-mail: g.f.schneider@lic.leidenuniv.nl. Phone: +31 71527 2700.

*E-mail: bouwman@chem.leidenuniv.nl. Phone: +31 71527 4550.

ORCID $\odot$

Elisabeth Bouwman: 0000-0001-7762-3968

\section{Author Contributions}

${ }^{\S}$ W.F. and T.F.D. contributed equally to this work. W.F. and T.F.D. designed and performed the experiments. G.F.S. and E.B. supervised the work. T.F.D. synthesized the copper complexes. W.F. fabricated the GFET devices. W.F. and T.F.D. performed the gas exposure experiments. L.M.C.L. performed the AFM and ellipsometry work. F.J. performed the DFT calculations. T.F.D., W.F., G.F.S., and E.B. wrote the manuscript. All the authors read and commented on the manuscript.

\section{Notes}

The authors declare no competing financial interest.

\section{ACKNOWLEDGMENTS}

This work is supported by NanoNextNL, a micro and nanotechnology consortium of the Government of The Netherlands and 130 partners. G.F.S. acknowledges financial support of the European Research Council under the European Union's Seventh Framework Programme (FP/2007-2013)/ ERC Grant Agreement 335879 project acronym "Biographene" and The Netherlands Organization for Scientific Research (NWO-VIDI 723.013.007). W.F. gratefully acknowledges financial support by The Netherlands Organization for Scientific Research (NWO-VENI 722.014.004) and the Swiss National Science Foundation (SNSF P300P2_154557). We thank Mr. Hans M. de Bruijn for synthesis of some of the copper compounds and Dr. Maxime A. Siegler (Johns Hopkins University, U.S.A.) for the determination of the crystal structure. We thank Arjmandi-Tash and Yanina Cesa for helpful discussions. We acknowledge Fons Lefeber and Karthick Sai Sankar Gupta for assistance with the NMR experiments. The gas exposure equipment was graciously provided by Jan-Kees Boerman from EMS B.V. Note: A patent application has been filed for the copper(I) hydridotrispyrazolylborate sensitized GFET devices.

\section{REFERENCES}

(1) Bakshi, A.; Shemansky, J.; Chang, C.; Binder, B. J. Plant Growth Regul. 2015, 34, 809-827.

(2) Gane, R. Nature 1934, 134, 1008.

(3) Keller, N.; Ducamp, M.-N.; Robert, D.; Keller, V. Chem. Rev. 2013, 113, 5029-5070.

(4) Esser, B.; Swager, T. M. Angew. Chem., Int. Ed. 2010, 49, 88728875 .

(5) Esser, B.; Schnorr, J. M.; Swager, T. M. Angew. Chem., Int. Ed. 2012, 51, 5752-5756.

(6) Zhang, A. Q.; Lieber, C. M. Chem. Rev. 2016, 116, 215-257.

(7) Kulkarni, G. S.; Reddy, K.; Zhong, Z. H.; Fan, X. D. Nat. Commun. 2014, 5, 4376.

(8) Fu, W.; Jiang, L.; van Geest, E. P.; Lima, L. M. C.; Schneider, G. F. Adv. Mater. 2017, 29, 1603610.

(9) Novoselov, K. S.; Geim, A. K.; Morozov, S. V.; Jiang, D.; Zhang, Y.; Dubonos, S. V.; Grigorieva, I. V.; Firsov, A. A. Science 2004, 306, 666-669.

(10) Arjmandi-Tash, H.; Belyaeva, L. A.; Schneider, G. F. Chem. Soc. Rev. 2016, 45, 476-493. 
(11) Schneider, G. F.; Kowalczyk, S. W.; Calado, V. E.; Pandraud, G.; Zandbergen, H. W.; Vandersypen, L. M. K.; Dekker, C. Nano Lett. 2010, 10, 3163-3167.

(12) Schedin, F.; Geim, A. K.; Morozov, S. V.; Hill, E. W.; Blake, P.; Katsnelson, M. I.; Novoselov, K. S. Nat. Mater. 2007, 6, 652-655.

(13) van Dijkman, T. F.; Siegler, M. A.; Bouwman, E. Dalton Trans. 2015, 44, 21109-21123.

(14) Chen, S.-Y.; Ho, P.-H.; Shiue, R.-J.; Chen, C.-W.; Wang, W.-H. Nano Lett. 2012, 12, 964-969.

(15) Das Sarma, S.; Adam, S.; Hwang, E. H.; Rossi, E. Rev. Mod. Phys. 2011, 83, 407-470.

(16) Wang, T.; et al. Nano-Micro Lett. 2016, 8, 95.

(17) Ferrari, A. C.; Meyer, J. C.; Scardaci, V.; Casiraghi, C.; Lazzeri, M.; Mauri, F.; Piscanec, S.; Jiang, D.; Novoselov, K. S.; Roth, S.; Geim, A. K. Phys. Rev. Lett. 2006, 97, 187401.

(18) Squires, T. M.; Messinger, R. J.; Manalis, S. R. Nat. Biotechnol. 2008, 26, 417-426.

(19) Szilagyi, P. A.; Westerwaal, R. J.; van de Krol, R.; Geerlings, H.; Dam, B. J. Mater. Chem. C 2013, 1, 8146-8155.

(20) Duan, X.; Li, Y.; Rajan, N. K.; Routenberg, D. A.; Modis, Y.; Reed, M. A. Nat. Nanotechnol. 2012, 7, 401-407.

(21) Reiner-Rozman, C.; Kotlowski, C.; Knoll, W. Biosensors 2016, 6, 17.

(22) Carrier, S. M.; Ruggiero, C. E.; Houser, R. P.; Tolman, W. B. Inorg. Chem. 1993, 32, 4889-4899.

(23) Hu, Z.; Williams, R. D.; Tran, D.; Spiro, T. G.; Gorun, S. M. J. Am. Chem. Soc. 2000, 122, 3556-3557.

(24) Muñoz-Molina, J. M.; Sameera, W. M. C.; Álvarez, E.; Maseras, F.; Belderrain, T. R.; Pérez, P. J. Inorg. Chem. 2011, 50, 2458-2467. (25) van Dijkman, T. F.; de Bruijn, H. M.; Brevé, T. G.; van Meijeren, B.; Siegler, M. A.; Bouwman, E. Dalton Trans. 2017, 46, 6433-6446.

(26) te Velde, G.; Bickelhaupt, F. M.; Baerends, E. J.; Fonseca Guerra, C.; van Gisbergen, S. J. A.; Snijders, J. G.; Ziegler, T. J. Comput. Chem. 2001, 22, 931-967.

(27) Li, X. S.; Cai, W. W.; An, J. H.; Kim, S.; Nah, J.; Yang, D. X.; Piner, R.; Velamakanni, A.; Jung, I.; Tutuc, E.; Banerjee, S. K.; Colombo, L.; Ruoff, R. S. Science 2009, 324, 1312-1314.

(28) Balandin, A. A. Nat. Nanotechnol. 2013, 8, 549-555. 